\title{
A review on influence of thermal studies of Nanomaterials
}

\author{
Suresh Sagadevan ${ }^{1, ~ *}$, B. Janarthanan ${ }^{2}$ \\ ${ }^{1}$ Department of Physics, Sree Sastha institute of Engineering and Technology, Chennai-600 123, India \\ ${ }^{2}$ Department of Automobile Engineering, Sree Sastha institute of Engineering and Technology, Chennai-600 123, India
}

Email address:

sureshsagadevan@gmail.com (S. Sagadevan)

\section{To cite this article:}

Suresh Sagadevan, B. Janarthanan. A Review on Influence of Thermal Studies of Nanomaterials. International Journal of Materials Science and Applications. Vol. 3, No. 6, 2014, pp. 370-377. doi: 10.11648/j.ijmsa.20140306.24

\begin{abstract}
Nanomaterials are defined as engineered materials with at least one dimension in the range of 1-100 nm. Particles of "nano" size have been shown to exhibit enhanced and novel properties including reactivity, greater sensing capability, and increased mechanical strength. The nanotechnique offers simple, clean, fast, efficient, and economic method for the synthesis of a variety of organic molecules, which has to provide the momentum for many chemists to switch from traditional method. To optimize the utilization of thermal conversion systems, it is essential to integrate them with thermal energy storage. In addition, study of the thermal properties of nanostructure materials against important grain growth is both scientific and technological interest. A sharp increase in grain size during consolidation of nanocrystalline powders to obtain full dense material may consequently result in the loss of some unique properties of nanocrystalline materials. The present review paper is aimed at understanding the thermal properties and its applications of nanostructure materials.
\end{abstract}

Keywords: Thermoelectric, Nanostructured Materials, Nanowires, Thermal Conductivity

\section{Introduction}

Nanotechnology is likely to have a profound impact on our economy and society and hence it may lead to the next industrial revolution. Because nanotechnology is essentially a set of techniques that allow manipulation of properties at a very small scale, it can have many applications, such as materials and manufacturing, electronics, medicine, energy and its environment, biotechnology, and information technology. From the beginning there was an issue in the thermal characterization of nanomaterials during their processing steps and in the applications, especially for the inorganic materials. Thermoanalytical and thermophysical testing methods are successfully applied for the determination of phase transitions, thermally induced chemical reactions and decompositions, gas adsorption and desorption studies, and thermal transport properties.

Nanomaterials are used as additives to enhance the properties of base materials. When it is added to the fluid, the produced mixture is denoted by nanofluid; while by adding the nanomaterials to phase change material (PCM), the produced mixture is denoted by nanocomposites. The different synthesis methods of preparation and the characterization of nanofluid, suspension, and the convection and conduction heat transfer in nanofluids have been reported by many authors [1-3]. Within the area of nanomaterials for thermal energy applications, many researchers have focused on the thermal conductivity effectiveness of nanofluids/composites which can improve their thermophysical properties [4-9]. In the last decade, nanostructured materials have been one of the most studied subjects, and nanotechnology applications are expected in practically all areas of science to produce higher performance products which will be faster, more sensitive and more accurate. Presently, a wide variety of synthesis procedures are available to obtain nanostructured materials [10-14], and material sizes, shapes, chemical compositions and consequently properties can vary by changing the process route. This paper critically reviews the existing studies dealing with the use of nanostructure materials. The aim of this paper is to demonstrate that the sensitivity of modern thermoanalytical methods are well suited with general applications on materials with a reduced particle size into the sub-micrometer range and that the results provide important parameters for the thermal characterization of nanomaterials. 


\section{Nanomaterials}

Nanotechnology is considered to be one of the most important future technologies involving several disciplines of science including solid state physics, solid state chemistry, solid state ionic, materials engineering, medical science and biotechnology. Manipulating matter at the nanometer scale, using building blocks with dimensions in the nano-size range, makes it possible to design and create new materials with unprecedented functionality and novel or improved properties. Nanostructured materials are made of nanosized grains or nanoparticles as building blocks, have a significant fraction of grain boundaries with a high degree of disorder of atoms along the grain boundaries (or particle surfaces), and a large ratio of interface (or surface) area to volume. Chemical composition of the phases and the interfaces, between nano-grains, must be controlled as well. One of the most important characteristics of nanostructured materials is the dependence of certain properties upon the size in nanoscale region. For example, electronic property, with quantum size effects, caused by spatial confinement of delocalized valence electrons, is directly dependent on the particle size. Small particle size permits conventional restrictions of phase equilibrium and kinetics to be overcome during the synthesis and processing by the combination of short diffusion distances and high driving forces of available large surfaces and interfaces. A wide range of materials, including metals and ceramics in crystalline, quasi-crystalline, or amorphous phases have been synthesized as nanosized or nanostructured materials.

World energy crisis has triggered more attention to energy saving and energy conversion systems with high efficiency. There is a growing awareness that nanoscience and nanotechnology can have a profound impact on energy generation, conversion, and recovery. Nanotechnologybased solutions are being developed for a wide range of energy problems such as, solar electricity, hydrogen generation and storage, batteries, fuel cells, heat pumps and thermoelectrics. Organic nanomaterials consist of fullerenes carbon nanotubes (CNT), single-walled carbon nanotubes (SWCNT), multi-walled carbon nanotubes (MWCNT), graphite and nanofibers. Most of the organic nanomaterials are carbon based nanomaterials [15]. While, metal and metal oxide based nanomaterials such as aluminium, zinc, copper, iron, aluminium oxide, iron oxide, titanium oxide are categorized as inorganic nanomaterials [16]. Quantum dots, such as $\mathrm{CdSe}, \mathrm{ZnS}, \mathrm{ZnO}$ etc are metalloid nanomaterials and are also categorized as inorganic nanomaterials $[17,18]$. Hybrid nanomaterials are the combination of organic - organic nanomaterials, organic inorganic nanomaterials and inorganic - inorganic nanomaterials through synthesis such as chemical vapor deposition (CVD), Electro spinning, atom transfer radical polymerization (ARTP) etc. [19,20].

\section{Nanostructure Materials for Thermoelectric Energy Conversion}

High performance thermoelectric materials in a wide range of temperatures are essential to broaden the application spectrum of thermoelectric devices. In addition the improvement of thermoelectric materials, device optimization is a crucial factor for efficient heat-to-electric power conversion and one of the key challenges is how to create a large temperature across a thermoelectric generator especially in the case of a dilute incident heat flux. A solarto-electric power conversion efficiency of $\sim 5 \%$ could be demonstrated. A solar thermoelectric generator with a large thermal concentration which minimizes the amount of thermoelectric nanostructure material shows great potential to enable cost-effective electrical power generation from the sun. The Seebeck effect can be exploited to generate power from a temperature difference. The efficiency of thermoelectric power generation systems depends on the material-specific thermoelectric figure of merit, the heat source and sink temperatures, and the heat transfer into and out of the devices. The efficiency of ideal thermoelectric devices $\left(\eta_{t e}\right)$ is determined by the operating temperatures and the materials' dimensionless figure of merit $(Z T)$, defined as $Z T=\left(S^{2} \sigma / k\right) T$, where $S, \sigma, k$, and $T$ are the Seebeck coefficient, electrical conductivity, thermal conductivity, and absolute temperature, respectively [21, 22]. The device efficiency can be expressed as:

$$
\eta_{t e}=\frac{T_{h}-T_{c}}{T_{h}} \frac{\sqrt{1+(Z T)_{M}}-1}{\sqrt{1+(Z T)_{M}}+\frac{T_{c}}{T_{h}}}
$$

where $T_{c}$ is the cold-side temperature, $T_{h}$ the hot-side temperature, and $(Z T)_{M}$ the effective $Z T$ of the thermoelectric materials between $T_{c}$ and $T_{h}$. According to Eq. (1), an efficiency of approximately $8.6 \%$ can be reached by imposing a temperature difference of $200{ }^{\circ} \mathrm{C}$ across an ideal thermoelectric device with $(Z T)_{M}=1$ and $T_{c}=20{ }^{\circ} \mathrm{C}$. In recent years, significant progress has been made on improving thermoelectric materials [23-28]. In addition the importance of the properties of thermoelectric materials for the device performance, the key for the application of thermoelectric generators is to establish a temperature difference across the device. Considering heat conduction only, the heat flux across a thermoelectric element with a length $L$ and temperature difference $\Delta T$ is given by $q=k \Delta T / L$ For $L=1 \mathrm{~mm}$ and thermal conductivity, $k=1 \mathrm{Wm}^{-1} \mathrm{~K}^{-1}$, and $\Delta T=100 \mathrm{~K}$, the heat flux is $105 \mathrm{~W} / \mathrm{m}^{2}$. Such high heat fluxes may result in large temperature drops outside the actual thermoelectric generator leading to a significant difference between the heat source/sink and thermoelectric junction temperatures. Thermal concentration uses heat conduction to concentrate the dilute heat flux from the sun $\left(\sim 1000 \mathrm{~W} / \mathrm{m}^{2}\right)$ to match the heat flux of thermoelectric devices, leading to a significant reduction in the materials required to fabricate solar thermoelectric generators. 


\section{Nanostructured Materials for Thermoelectric}

Thermoelectrics are considered those materials have low thermal conductivity with a large electrical conductivity. Low dimensional materials are high efficient thermoelectrics because it can become thermal insulators with high electrical conductivities. Thermal insulation is widely present in chemical plants (e. g. pipes, furnaces, etc.), so thermoelectric materials could replace current insulations to harvest that thermal energy and transform it into electricity. Thermoelectric devices can play a very important role in clean energy generation, conversion and recovery. Thermoelectrics are 'fuel-free' purely solid-state devices with no moving parts and therefore are extremely reliable. Thermoelectrics can harvest residual low-grade energy which otherwise is wasted. Next generation thermoelectric devices shall certainly revolutionize several concepts of energy harvesting and conversion for power generation, refrigeration/heating, and thermal sensing both in terrestrial and space applications. The key factor for improving the performance of thermoelectric devices can be through the nanotechnology development of thermoelectric materials with novel composition, low dimensionality, and advanced architectures. Another approach for improving the performance of thermoelectric materials is through nanostructuring. A Nanocrystalline material with grain size is in the nanometer regime which have significant amount of grain boundaries. Grain boundaries are more selective for the diffraction of phonon as compare to that of electrons. Thermoelectic materials convert heat directly into electricity (and vice versa) and can thus recycle some of the energy contained in, for instance, hot exhaust streams. While low efficiency has traditionally limited the use of thermoelectrics to niche markets, recently developed nanostructured thermoelectrics, with much better performance than bulk thermoelectrics, mark the beginning of a new era. Progress has also been made towards inexpensive, large-scale production methods. Beyond transport and industrial production, interesting application areas include the transformation of low-grade solar thermal or geothermal energy, or the use of human body heat to power portable electronics.

\section{Thermal Concept of Nanomaterials}

A number of methods, from first principle calculations to empirical models, were applied to understand the metastable phase formation in nanocrystalline materials. The fundamental difference between coarse grain and nanocrystalline materials lies in the formation of large volume fraction of interfaces in nanocrysttaline materials that has significant influence on the thermodynamics of phase formation/transformation. In the bulk state, the thermodynamic condition for phase $\alpha$ to be stable over metastable phase $\beta$ is that $\mathrm{G}_{\alpha}<\mathrm{G}_{\beta}$. In case of nanocrystalline materials, the contribution of interfacial energy term $\left(G_{\text {int }}\right)$ to the free energy cannot be neglected as in coarse-grained polycrystalline materials. The interfacial free energy of nanocrystalline materials, can be expressed as:

$$
\Delta G_{\text {int }}=3 \gamma_{\text {int }} V_{m} / d
$$

Where $\mathrm{V}_{\mathrm{m}}$ is the molar and $\mathrm{d}$ is the crystallite size. In the nanocrystalline state, $\beta$ may become stable if $\left.G_{\alpha}+G_{\alpha}^{\mathrm{int}}\right\rangle G_{\beta}+G_{\beta}^{\mathrm{int}}$. In other words, if the rate of increase of total free energy $\left(\mathrm{G}+\mathrm{G}_{\text {int }}\right)$ with decrease in crystallite size of $\alpha$ phase is higher than that of $\beta$ phase, the latter will become stable over the former below a critical crystallite size.

\subsection{Melting Behavior of Nanomaterials}

The melting of a dispersed nanosized metal in a metallic matrix can be determined precisely also at very small quantity level. Differential Scanning Calorimetry (DSC) can demonstrate the change of melting temperatures depending on grain sizes.

$$
\Delta T_{m}=T_{m}(b)-T_{m}(r)=\frac{2 T_{m}(b) \sigma_{s l}}{\Delta H_{f}(b) \rho_{s} r}
$$

Where $T_{m}(b), \Delta H_{f}(b)$ and $\rho_{s}$ are the bulk melting temperature, the bulk latent heat of fusion, and the solid phase density, respectively. $r$ represents the radius of a spherical particle, and $T_{m}(r)$ is the melting point of a particle with radius $r, \sigma_{s l}$ is the solid-liquid interfacial energy. The Gibbs-Thomson equation predicts a linear relationship between the melting point depression and the inverse of the particle size.

\section{Nanostructured Materials for Solar Thermoelectric Generators}

One of the novel uses of thermoelectric nanostructured materials is in the field of solar thermoelectric generators. Solar thermoelectric generators are devices that convert the heat from sunlight into electrical energy. Solar thermoelectric generators allow more efficient use of the solar energy because it captures a wide solar spectrum which is not possible for photovoltaic cells [29, 30]. A solar thermoelectric generator is made up of several elements: thermoelectric materials, heat absorption element, metal interconnects, and substrates. Such devices use the traditional $\mathrm{p}$ and n-type semiconductor junctions with high thermoelectric efficiency materials such as SiGe alloy [30] and $\mathrm{Bi}_{2} \mathrm{Te}_{3}$ [31]. However, the efficiency of solar thermoelectric generator units is about $1 \%$ which is low compared to the $11 \%$ for silicon-based photovoltaic cells [31, 32]. New thermoelectric materials and harvesting techniques have been developed to overcome efficiency barriers, increase absorbed solar energy, and reduce radiation losses $[29,31]$. Current developments are also focused on materials that can provide higher operating temperatures and higher thermoelectric efficiencies with efficiencies close to $4.6 \%$ 
$[32,33]$. The thermoelectric energy efficiency can be quantified by the dimensionless thermoelectric figure of merit $\left(Z T=S^{2} \sigma T / K\right.$, where $S, \sigma, T$ and $K$ are the Seebeck coefficient, electrical conductivity, absolute temperature, and thermal conductivity respectively) [34]. Materials with large $\sigma$ and low $K$ (electron-crystal, phonon-glass materials) are desirable to obtain a high $Z T$, so nanostructured materials are perhaps the best option for high efficient solar thermoelectric generator. Another important parameter to take into consideration is the thermoelectric power $\left(S^{2} \sigma\right)$ which strongly dependent on the $\sigma$ ( $S$ is inversely proportional to the $\sigma)$. Nanostructuring could affect $S^{2} \sigma$ due to the influence of surface state effect [35], or quantum confinement [36]. Sample preparation and crystalline quality are also factors that contribute to the good quality of thermoelectric materials. For example, the thermoelectric characteristics of n-type $\mathrm{Bi}_{2} \mathrm{Te}_{3}$ nanowire bundles (nanowire diameter approx. $70 \mathrm{~nm}$ ). It was found that $S^{2} \sigma$ is about $6.2 \times 10^{-4} \mathrm{~W} / \mathrm{m}-\mathrm{K}^{2}$ at $300 \mathrm{~K}$ which is a factor of 10 larger than the value reported for $n$ type $\mathrm{Bi}_{2} \mathrm{Te}_{3}$ single nanowires [37] and a factor of 3 smaller than nanocrystalline $\mathrm{Bi}_{2} \mathrm{Te}_{3}$ [38] (all these power factors were measured at similar $\sigma$ ).

\section{Thermoelectric Nanowire Sensors}

Rapid and highly sensitive detection of chemical and biological species is critical in many fields such as medical treatment, monitoring of toxic agents, and safety. The use of nanostructured materials for those applications has led to the development of a series of impressive sensor technologies. The large impact of nanosensors can be understood by the broad number of applications ranging from the detection of transmembrane pore-forming proteins by silicon nanowires, [39] to chemical sensing by $\mathrm{ZnO}$ nanoribbons [40], to the sensing of biomolecules by silver triangular nanoparticles [41]. Some of the reasons for such interest are: the large density of sensing devices in a small area with different functionalities given their small dimensions [42, 43]; the mass transfer rate of target species from solution to the surface of the nanosensor is not a limiting process (contrary to their bulk counterpart); and the ability to detect small concentration of target species. For example, silicon field effect transistor nanowire sensors have been able to detect target species to the picograms/ml level [44]. Generally, nanosensors are divided into 2 groups: the electrical and optical based sensing approaches. For the electrical based nanosensors, the sensing approach includes measuring electrical changes of nanostructured materials due to chemical, biochemical, or cell activity events. For optical based nanosensors, the sensing event will trigger an optical response, which could be either a change of the fluorescence intensity (also known as fluorescent tagged approach), or a change of a material's property (e. g. refractive index) known as label free approach [45]. Despite these considerable advantages, optical methods present limitations such as photo bleaching, interaction of non-target species in solution with the fluorescent probes, and inability to be used in turbid solutions. On the other hand, electrical based sensors overcome those limitations, but it also presents a series of disadvantages such as electro interference with the sensing media, and electrochemical reaction of non-target species in solution. Thermoelectric sensors are present in a number of aquatic animals. For example, the extraordinary ability to sense temperature changes and small electric fields of pray by sharks is carried out by a thermoelectric gel in the “ampullae of Lorenzini”[46]. Thermoelectric sensor response would be based on changes of carrier density due to surface binding events, so small diameter nanowires with large mobilities are expected to improve signal to noise ratio.

\section{Thermal Analysis of Nanocrystalline Materials}

Thermal analysis of nanocrystalline materials is used to study the phase transitions of nanoparticles, which are expected to be much different from bulk crystals. Many workers [47, 48] reported the phase transition studies of nanostructured materials. The free energy of nanoparticles has been reported to be higher [49] than that of their polycrystalline counterparts. The changes occurred on their microstructure and atomic configuration can well study when they are subjected to thermal analysis. Many authors [50-53] observed new phases and additional exothermic and endothermic peaks in nanostructured materials, which were not present in their bulk counterparts. The new peaks and phases are explained on the basis of grain growth process and their microstructure and atomic configuration changes when exposed to high temperature. A large fraction of atoms in nanoparticles are surface atoms and these atoms have significant influence on the thermal properties of nanostructure materials. Structural changes can be expected in small particles because of the large amount of free energy [49] associated with their grain boundaries. The energy associated with the interface regions becomes substantial when the size of the crystallites is in nanosized regime and it may influence phase transitions in nanophase materials. Other types of lattice imperfections may also exist due to small size of the particles. Hence the phase transitions in nanoparticles are expected to exhibit modified behaviour from that of the bulk materials.

The advances of nanotechnologies in the past decades have resulted in the burst of promising synthesis, processing and characterization technologies, which enables the routine production of a variety of nanomaterials with highly controlled structures and related properties. By controlling the structures of nanomaterials at nanoscale dimensions, the properties of the nanostructures can be controlled and tailored in a very predictable manner to meet the needs for a variety of applications. Examples of the engineered nanostructures include metallic and non-metallic nanoparticles, nanotubes, quantum dots and super lattices, thin films, nanocomposites and nanoelectronic and optoelectronic devices which utilize the superior properties 
of the nanomaterials to fulfill the applications. Many properties of the nanoscale materials have been well studied, including the optical electrical, magnetic and mechanical properties. However, the thermal properties of nanomaterials have only seen slower progresses. This is partially due to the difficulties of experimentally measuring and controlling the thermal transport in nanoscale dimensions. Atomic Force Microscope (AFM) has been introduced to measure the thermal transport of nanostructures with nanometer-scale high spatial resolution, providing a promising way to probe the thermal properties with nanostructures [54]. Moreover, the theoretical simulations and analysis and of thermal transport in nanostructures are still in infancy. Available approaches including numerical solutions of Fourier's law, computational calculation based on Boltzmann transport equation and Molecular-dynamics (MD) simulation, all have their limitations [54]. More importantly, as the dimensions go down into nanoscale, the availability of the definition of temperature is in question. In non-metallic material system, the thermal energy is mainly carried by phonos, which have a wide variation in frequency and the mean free paths (mfp). The heat carrying photons often have large wave vectors and mean free path in the order of nanometer range at room temperature, so that the dimensions of the nanostructures are comparable to the mean free path and wavelengths of photons. However the general definition of temperature is based on the average energy of a material system in equilibrium. For macroscopic systems, the dimension is large enough to define a local temperature in each region within the materials and this local temperature will vary from region to region, so that one can study the thermal transport properties of the materials based on certain temperature distributions of the materials. But for nanomaterial systems, the dimensions may be too small to define a local temperature. Moreover, it is also problematic to use the concept of temperature which is defined in equilibrium conditions, for the non-equilibrium processes of thermal transport in nanomaterials, posing difficulties for theoretical analysis of thermal transport in nanoscale [54].

Recent advances in experiments have shown that certain nanomaterials have extraordinarily thermal properties compared to their macroscopic counterparts, as will be explained in detail in this section. In nanomaterials systems, several factors such as the small size, the special shape, the large interfaces modified the thermal properties of the nanomaterials, rendering them the quite different behavior as compared to the macroscopic materials. As mentioned above, as the dimension goes down to nanoscales, the size of the nanomaterials is comparable to the wavelength and the mean free path of the photons, so that the photon transport within the materials will be changed significantly due the photon confinement and quantization of photon transport, resulting in modified thermal properties. For example, nanowires from silicon have smaller thermal conductivities compared to bulk silicon [55]. The special structure of nanomaterials also affects the thermal properties. For example, because of it tubular structures of carbon nanotubes, they have extreme high thermal conductivity in axial directions, leaving high anisotropy in the heat transport in the materials [56]. The interfaces are also very important factor for determination of the thermal properties of nanomaterials. Generally, the internal interfaces impede the flow of heat due to photon scattering. At interface or grain boundary between similar materials, the interface disorder scatters phonons, while as the differences in elastic properties and densities of vibration states affect the transfer of vibration energy across interfaces between dissimilar materials. As a result, the nanomaterials structures with high interfaces densities would reduce the thermal conductivity of the materials [57]. These interconnected factors had joined together to determine the special thermal properties of the nanomaterials.

\section{Thermal Stability of Carbon Nanotubes}

The stiff $s p 3$ bonds in diamond structure result in high phonon speed and consequently high thermal conductivities of the material. In carbon nanotubes, the carbon atoms are held together by the even stronger $s p 2$ bonds, so that the nanotube structures, consisting of seamlessly joined graphitic cylinders are expected to have extraordinarily high thermal conductivities. The rigidity of the these nanotubes, combined with virtual absence of atomic defects or coupling to soft photon modes of the embedding medium, should make isolated nanotubes very good candidates for efficient thermal conductors [58]. The high thermal conductivity of carbon nanotubes were first proved by some theoretical calculations $[58,59]$. Savas Berber et al combined the equilibrium and nonequilibrium molecular dynamics simulations with Tersoff potential to determine the thermal conductivity of carbon nanotubes and its dependence on temperature. Recently Motoo Fujii et al measured the thermal conductivity of single carbon nanotube using a suspended sample-attached T-type nanosensor [60], and reported values exceeding $2000 \mathrm{~W} / \mathrm{mK}$ for a carbon nanotube of $9.8 \mathrm{~nm}$ diameter. These experimental values were in the range of the theoretical calculations, which provides high thermal conductivity of the carbon nanotubes experimentally. Due to their high thermal conductivities, carbon nanotubes or nanotubes based nanocomposite could be promising candidates for heat transport management in many applications such as in the integrated circuits, optoelectronic devices and MEMS structures.

Carbon nanotube (CNT) samples show significant difference in their thermal stability under oxidative atmosphere, depending on their preparation conditions. The two samples tested reveal the same oxidizable carbon content of $92.67 \%$, which burns out in the temperature range 400 to $750{ }^{\circ} \mathrm{C}$, but the volatile content before start of oxidation is 10 times higher in the modified sample, and the residue at $1000{ }^{\circ} \mathrm{C}$ (ash) is 3 times less in the modified CNT sample. The oxidation range of this CNT samples is much lower compared with known ranges for bulk graphite and diamond 
samples. Though carbon atoms are involved in aromatic rings like for graphite, the $\mathrm{C}=\mathrm{C}$ bond angles are no longer planar in the CNTs and the $\mathrm{C}-\mathrm{C}$ bond length is actually elongated by the curvature imposed.

\section{Thermal Properties of Nanowires}

One-dimensional nanowires may offer ultra low thermal conductivities, quite different from that of carbon nanotubes. In nanowires, phonons behave differently from those in the corresponding bulk materials due to the quantum confinement in the one dimension structures. The nanowire surface can introduce surface phonon modes, resulting in many different phonon polarizations other than the two transverse and one longitudinal acoustic branch found in bulk semiconductors. Those changes in the dispersion relation can modify the group velocity and the density of states of each branch. The phonon lifetime also changes due to the strong phonon- phonon interactions and the boundary scattering within the nanostructures [54]. Thus the phonon transports and the thermal properties of the nanowires will be significantly different from that of the bulk materials. The phonon transport of semiconducting nanowires have been studied experimentally [55, 61] and theoretically [62-64]. Deyu et al measured the thermal conductivity of silicon nanowires using a micro fabricated suspended device over a temperature range of $20-320 \mathrm{~K}[54,61]$. Although the nanowires had well-defined crystalline order as in bulk materials, the observed thermal conductivity was more than two orders of magnitude smaller than that of bulk silicon, which also showed a strong dependence on the nanowires size. For a silicon nanowire with $22 \mathrm{~nm}$ diameter, the thermal conductivity was reduced to be in the sub $10 \mathrm{~W} / \mathrm{m}$ range. The appreciable change of the thermal conductivity compared to the bulk materials was ascribed to phonon-boundary scattering and the possible change in phonon dispersion due to confinement within the nanostructures.

The same research group also measured the thermal conductivity on $\mathrm{Si} / \mathrm{SiGe}$ super lattice nanowires [61], with even smaller thermal conductivity reported on the super lattice nanowires compared to pure silicon nanowires. These experimental results agreed fairly well with the theoretical calculations based on phonon dispersions approach [62, 63]. The low thermal conductivity in these one dimensional semiconducting nanowires will find applications in thermoelectric power generation and the thermoelectric refrigeration. However, they are not desired in for thermal management in electronics, which generally need much higher thermal transport capabilities. Besides the one dimensional nanowires, multilayer and superlattices are another type of nanostructures offering low thermal conductance. David G. Cahilla et al wrote an excellent review about the thermal transport of the nanomaterials and elaborated the thermal transport in multilayer and superlattices [54]. Multilayers and superlattices are thin film structures that contain alternating layers of two different materials stacked upon each other. In multilayers structure the films can be either amorphous or polycrystalline while in superlattices the films are single crystal. There are many effects in the multilayers or superlattices structures that affect the phonon transport properties of the materials. When alternating layers of materials are stacked together, many collective mode of phonon transport may appear besides the phonon modes in each single layer. This stacking effect will be more apparent when the coherence phonon length scales are much larger than that of single layer. It is also a coupled behavior resulting from interference of phonon waves reflected from multiple interfaces. When the mean free path of phonons span multiple interfaces, the phonon dispersion relation is modified and zone folding occurs, even resulting in multiple phonon band gaps [65]. Moreover, due to the modification of the phonon dispersion, the phonon group velocities will be reduced significantly and the scattering rate will also be increased. The interfaces is also an important factor in determine the phonon transport properties due to the high density of interfaces in the multilayers or superlattices structures. For example, if the two materials in the super lattice have large mismatch in the phonon dispersion relations, phonons in certain frequency range cannot propagate to the neighboring layer unless there are mode conversions at the interface; The interfaces between two different materials with different lattice constants can contain dislocations and defects, which can also scatter phonons and reduce thermal conductivity; physical roughness and alloying will also exist at the interface depending on the processing, and affect the phonon transport. The overall effect in these factors on the phonon transport is the multilayer and super lattice structures are a general decrease of thermal conductivities.

\section{Conclusion}

Nanostructured materials are becoming the materials of choice for thermoelectric applications mostly due to the reduction of the thermal conductivity without a negative influence on the electronic properties. In spite of the large improvements of the thermoelectric efficiency by nanostructuring, $Z T$ values are still not high enough for a wide spread use of thermoelectrics. However, a large number of applications could presently benefit from nanostructured thermoelectric. Engineers in collaboration with materials scientists would make possible to expand the horizon of applications for thermoelectric nanostructured materials.

\section{Acknowledgement}

We extend our sincere thanks to the management of Sree Sastha Institute of Engineering and Technology, Chembarambakkam, Chennai-600123, India for their encouragements and support provided for this work. 


\section{References}

[1] Ghadimi, A.; Saidur, R.; Metselaar, H.S.C. A review of nanofluid stability properties and characterization in stationary conditions.Int. J. Heat Mass Tran., 2011, 54, 4051-4068.

[2] Saidur, R.; Leong, K.Y.; Mohammad, H.A. A review on applications and challenges of nanofluids. Renew. Sust. Energ. Rev., 2011, 15, 1646-1668.

[3] Haddad, Z.; Oztop, H.F.; Abu-Nada, E. A review on natural convective heat transfer of nanofluids. Renew. Sust. Energ. Rev., 2012, 16, 5363-5378.

[4] Taylori, R.; Coulombe, S.; Otanicar, T. Critical review of the novel applications and uses of nanofluids, Proceedings of the $3^{\text {rd }}$ International Conference on Micro/Nanoscale Heat \& Mass Transfer, Atlanta, GA, March, Anonymous , 2012, 3-6.

[5] Kandlikar, S.G. History, advances, and challenges in liquid flow and flow boiling heat transfer in microchannels: a critical review. J. Heat Trans-T ASME, 2012, 134, 034001.

[6] Mahian, O.; Kianifar, A.; Kalogirou, S.A. A review of the applications of nanofluids in solar energy. Int. J. Heat Mass Tran., 2013, 57(2), 582-594.

[7] Sureshkumar, R.; Mohideen, S.T.; Nethaji, N. Heat transfer characteristics of nanofluids in heat pipes: a review. Renew. Sust.Energ. Rev., 2013, 20, 397-410.

[8] Barber, J.; Brutin, D.; Tadrist, L. A review on boiling heat transfer enhancement with nanofluids. Nanoscale Res. Lett., 2011, 6(1), 1-16.

[9] Fan, J.; Wang, L. Review of heat conduction in nanofluids. $J$. Heat Transf., 2011, 133(4), 133-138

[10] Morales A.M, Lieber C. M, Science 279: 208(1998)

[11] Wang S R, Huang J, Zhao Y Q, Mater Lett 60:1706(2006)

[12] Xu C K, Xu G D, Liu Y K, Scr Mat 46:789(2002)

[13] Gotoh Y, Fujimura K, Koike M, Mater Res Bull 36:2263(2001)

[14] Zhu H L, Ji X, Yang D R, J Mater Sci 41:3489(2006)

[15] Scida, K.; Stege, P.W.; Haby, G.; Messina, G.A.; Garcia, C.D. Recent application of carbon-based nanomaterials in analytical chemistry: critical review. Anal. Chim. Acta, 2011, 691, 6-17.

[16] Dreizin, E.L. Metal-based reactive nanomaterials. Prog. Energ. Combust., 2009, 35, 141-167.

[17] Singh, S.C.; Ram, G. Drop shape zinc oxide quantum dots and their self-assembly into dendritic nanostructure: liquid assisted pulsed lase ablation and characterizations. Appl. Surf. Sci., 2012, 258,2211-2218.

[18] Gong, S.; Yang, G.; Ban, D.; Fu, J.; Fu, Y. Three-pulsed photon echo induced by the optical transitions of excitonsin core-shell $\mathrm{CdSe} / \mathrm{ZnS}$ nanocrystal quantum dots. Opt. Mater., 2011, 34, 36-41.

[19] Sebaa, M.; Nguyen, T.Y.; Paul, R.K.; Mulchandani, A.; Liu, H. Graphene and carbon nanotubes-graphene hybrid nanomaterials for human embryonic stem cell culture. Mater. Lett., 2013, 92, 122-125.
[20] Yuan, J.; Muller, A.H.E. One-dimensional organic-inorganic hybrid nanomaterials. Polymer, 2010, 51, 4015-4036

[21] Goldsmid, H. J., Thermoelectric Refrigeration. Plenum, New York, (1964).

[22] Rowe, D. M. Thermoelectrics Handbook Nano to Macro, CRC Taylor \& Francis, Boca Raton, (2006).

[23] Hsu, K. F., Loo, S., Guo, F., Chen, W., Dyck, J. S.“Cubic $\mathrm{AgPb}(\mathrm{m}) \mathrm{SbTe}(2+\mathrm{m})$ : bulk thermoelectric materials with high figure of merit", Science, 303, 818-821 (2004).

[24] Dresselhaus, M. S., Chen, G., Tang, M. Y., Yang, R. G., Lee, H., et al., "New Directions for Low-Dimensional Thermoelectric Materials", Advanced Materials, 19, 10431053, (2007).

[25] Poudel, B., Hao, Q., Ma, Y., Lan, Y., Minnich, A."HighThermoelectric Performance of Nanostructured Bismuth Antimony Telluride Bulk Alloys", Science, 320, 634-638 (2008).

[26] Heremans, J. P., Jovovic, V., Toberer, E. S., Saramat, A., Kurosaki, K., et al., "Enhancement of Thermoelectric Efficiency in PbTe by Distortion of the Electronic Density of States", Science, 321, 554-557, (2008).

[27] Tritt, T. M., Subramanian, M. A., "Thermoelectric Materials, Phenomena, and Applications: A Bird's Eye View", MRS Bulletin, 31, 188-198, (2006).

[28] Minnich, A., Dresselhaus, M. S., Ren, Z. F., Chen, G., "Bulk nanostructured thermoelectric materials: current research and future prospects", Energy \& Environmental Science, 2, 466479, (2009).

[29] Kraemer D, Poudel B, Feng HP, Caylor JC, Yu B, Yan X, et al. High-performance flat-panel solar thermoelectric generators with high thermal concentration. 2011; Nat Mater.10: 532-538.

[30] Fuschillo N, Gibson R. Germanium-silicon, lead telluride, and bismuth telluride alloy solar thermoelectric generators for venus and mercury probes. Advanced Energy Conversion. 1967; 7: 43-52.

[31] Amatya R, Ram RJ. Solar Thermoelectric Generator for Micropower Applications. Journal of Electronic Materials. 2010; 39: 1735-1740.

[32] Kraemer D, Poudel B, Feng HP, Caylor JC, Yu B, Yan X. High-performance flat-panel solar thermoelectric generators with high thermal concentration. 2011; Nat Mater.10: 532538.

[33] Baranowski LL, Snyder GJ, Toberer E.S. Concentrated solar thermoelectric generators. Energy \& Environmental Science. 2012; 5: 9055-9067.

[34] Tritt TM. Thermoelectric Phenomena, Materials, and Applications. Annual Review of Materials Research. 2011; 41: 433-448.

[35] Martinez JA, Cho JH, Liu XH, Luk TS, Huang JY, Picraux ST, et al. Contribution of radial dopant concentration to the thermoelectric properties of core-shell nanowires. Applied Physics Letters. 2013; 102: 103101.

[36] Cornett JE, Rabin O. Thermoelectric figure of merit calculations for semiconducting nanowires. Applied Physics Letters. 2011; 98: 182104. 
[37] Zhou JH, Jin CG, Seol JH, Li XG, Shi L. Thermoelectric properties of individual electrodeposited bismuth telluride nanowires. Applied Physics Letters. 2005; 87: 133109.

[38] Takashiri M, Takiishi M, Tanaka S, Miyazaki K, Tsukamoto H. Thermoelectric properties of n-type nanocrystalline bismuthtelluride-based thin films deposited by flash evaporation. Journal of Applied Physics. 2007; 101: 074301.

[39] Martinez JA, Misra N, Wang Y, Stroeve P, Grigoropoulos CP, Noy A. Highly efficient biocompatible single silicon nanowire electrodes with functional biological pore channels. 2009; Nano Lett.9: 1121-1126.

[40] Fan ZY, Wang DW, Chang PC, Tseng WY, Lu JG. ZnO nanowire field-effect transistor and oxygen sensing property. Applied Physics Letters. 2004; 85: 5923-5925.

[41] McFarland AD, Van Duyne RP. Single silver nanoparticles as real-time optical sensors with zeptomole sensitivity. Nano Letters. 2003; 3: 1057-1062.

[42] Cui Y, Wei Q, Park H, Lieber CM. Nanowire nanosensors for highly sensitive and selective detection of biological and chemical species. 2001; Science.293: 1289-1292.

[43] Misra N, Martinez JA, Huang SCJ, Wang YM, Stroeve P, Grigoropoulos CP, et al. Bioelectronic silicon nanowire devices using functional membrane proteins. Proceedings of the National Academy of Sciences of the United States of America. 2009; 106: 13780-13784.

[44] Zheng G, Patolsky F, Cui Y, Wang WU, Lieber CM. Multiplexed electrical detection of cancer markers with nanowire sensor arrays. 2005; Nat Biotechnol.23: 1294-1301.

[45] Fan X, White IM, Shopova SI, Zhu H, Suter JD, Sun Y. Sensitive optical biosensors for unlabeled targets: a review. 2008; Anal Chim Acta.620: 8-26.

[46] Brown BR. Neurophysiology: Sensing temperature without ion channels. Nature. 2008; 454: 246-246.

[47] W Chang, F Cosandey \& H Hahn, Nanostructured Materials 2,29 (1993)

[48] R Nitche, M Rodewald, G Skandan, H Fuess \& H Hahn, Nanostructured Materials 7,535 (1996)
[49] X Y Qin, X J Wu \& L F Cheng, Nanostructured Materials $2,99(1993)$

[50] A Cziraki, Zs.Tonkovies, I Geores, B Fogarassy, I Groma, D Toth-Kadar, T Tamoczi \& I Bakonyi, Ma Sci Engg. A 180,531 (1994)

[51] D A Keen \& S Shull, J. Phys. Condensed Matter 7,5793 (1995)

[52] H Gleiter, Prog. Mater. Sci. 53, 223 (1989)

[53] K W Browall, J S Kasper \& H Widemeier, J. Solid State Chem. $10,20(1974$

[54] David G C, Wayne K F, Kenneth E G, Gerald D M, Arun M, Humphrey J M, Roberto M and Simon R P 2003 Journal of Applied Physics 93793

[55] Deyu L, Yiying W, Philip K, Li S, Peidong Y and Arun M 2003 Applied Physics Letters 832934

[56] Dresselhaus M S, Dresselhaus G and Avouris P 2001 Carbon Nanotubes Synthesis, Structure, Properties, and Applications (Berlin: Springer)

[57] Costescu R M, Cahill D G, Fabreguette F H, Sechrist Z A and George S M 2004 Science 303989

[58] Berber S, Kwon Y-K and Tománek D 2000 Physical Review Letters 844613

[59] Osman M A and Srivastava D 2001 Nanotechnology 21

[60] Fujii M, Zhang X, Xie H, Ago H, Takahashi K, Ikuta T, Abe H and Shimizu T 2005 Physical Review Letters 95065502

[61] Deyu L, Yiying W, Rong F, Peidong Y and Arun M 2003 Applied Physics Letters 833186

[62] Mingo N, Yang L, Li D and Majumdar A 2003 Nano Lett. 3 1713

[63] Mingo N 2003 Physical Review B (Condensed Matter and Materials Physics) 68113308

[64] Jie Z and Alexander B 2001 Journal of Applied Physics 89 2932

[65] Simkin M V and Mahan G D 2000 Physical Review Letters 84 927 